\title{
NOTES
}

\section{Syntheses of Perfluoroalkylated Styrene Monomers with Bis(perfluoroalkanoyl) Peroxides}

\author{
Motohiro Mitani, Hideo Sawada, Masaharu Nakayama, \\ Masato Yoshida, * and Nobumasa Kamigata* \\ Tsukuba Research Laboratory, Nippon Oil \& Fats Co., Ltd., \\ Tokodai, Tsukuba, Ibaraki 300-26, Japan \\ *Department of Chemistry, Faculty of Science, Tokyo Metropolitan University, \\ Fukazawa, Setagaya-ku, Tokyo 158, Japan
}

(Received January 4, 1990)

\begin{abstract}
KEY WORDS Perfluoroalkylation / Perfluoroalkylpolystyrene Precursor / Bis(perfluoroalkanoyl) Peroxides /
\end{abstract}

Recently, there has been great interest in perfluoroalkylated polymers due to their unique properties, such as water- and oilrepellency, and low friction. In particular, polystyrenes possessing long perfluoroalkyl chains in the aromatic nuclei have extremely low critical surface tension and high solubility in organic solvents. ${ }^{1}$ Therefore, it is important to develop convenient methods for the syntheses of perfluoroalkylated styrene monomers. To our knowledge, there have been only few examples for the syntheses of styrene monomers having perfluoroalkyl groups in the aromatic nuclei: e.g., p-perfluoroalkylstyrene was synthesized from $p$-perfluoroalkylated acetophenone prepared by the reaction of perfluoroalkyl iodide with $p$-bromoacetophenone in the presence of copper catalyst in three steps. ${ }^{2}$ We have studied the perfluoroalkylation of aromatic and heteroaromatic compounds with bis(perfluoroalkanoyl) peroxides $\left[\left(\mathrm{R}_{\mathrm{F}} \mathrm{CO}_{2}\right)_{2}: \quad \mathrm{R}_{\mathrm{F}}=\mathrm{C}_{3} \mathrm{~F}_{7} \quad(\mathbf{1 a}), \mathrm{C}_{6} \mathrm{~F}_{13}\right.$ (1b) ]. ${ }^{3}$ However, on treatment of styrene with 1a, the addition of $\mathbf{1 a}$ on vinyl group of the styrene occurred with evolution of $\mathrm{CO}_{2}$, and perfluoropropylated styrene monomer could not be obtained. ${ }^{4}$ We investigated the perfluoroalkylation of ethylbenzene derivatives with 1 and tried to convert the products into perfluoroalkylated styrenes, and the results are presented in this paper.

\section{RESULTS AND DISCUSSION}

The reaction of $\alpha$-chloroethylbenzene (2a) with $1 \mathrm{a}$ in Freon 113 solution afforded perfluoropropylated $\alpha$-chloroethylbenzene (3a) in $61 \%$ isolated yield. ${ }^{5}$

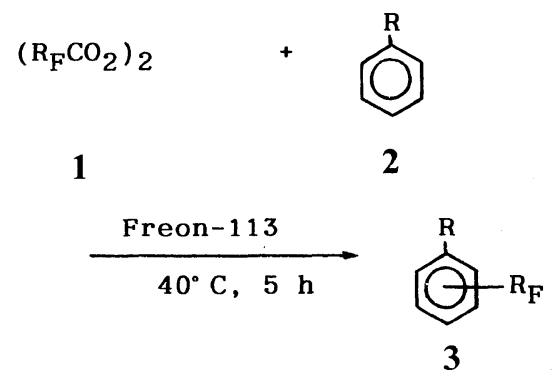

Similarly, $\beta$-chloroethylbenzene (2b) was reacted with $1 \mathrm{a}$ to give perfluoroalkylated $\beta$ chloroethylbenzene (3b) in good yield. Perfluorohexylation of $\mathbf{2 b}$ with $\mathbf{1 b}$ also proceeded in good yield. $\beta$-Bromoethylbenzene was perfluoropropylated in $41 \%$ yield under similar conditions. However, $\alpha$-bromoethylbenzene did not give the expected 3c. In the 
Table I. Perfluoroalkylations of ethylbenzene derivatives with bis(perfluoroalkanoyl) peroxides

\begin{tabular}{|c|c|c|c|c|}
\hline $\mathrm{R}$ in $\mathbf{2}$ & & $\mathbf{R}_{\mathrm{F}}$ in $\mathbf{1}$ & Products & Yield $/ \%^{\mathrm{a}}$ \\
\hline $\mathrm{CH}(\mathrm{Cl}) \mathrm{CH}_{3}$ & $(\mathbf{2 a})$ & $\mathrm{C}_{3} \mathrm{~F}_{7}$ & $3 \mathbf{a}$ & $61^{b}$ \\
\hline $\mathrm{CH}_{2} \mathrm{CH}_{2} \mathrm{Cl}$ & $(\mathbf{2 b})$ & $\mathrm{C}_{3} \mathrm{~F}_{7}$ & 3b & $69^{b}$ \\
\hline $\mathrm{CH}(\mathrm{Br}) \mathrm{CH}_{3}$ & $(2 \mathrm{c})$ & $\mathrm{C}_{3} \mathrm{~F}_{7}$ & $3 c$ & None $^{c}$ \\
\hline $\mathrm{CH}_{2} \mathrm{CH}_{2} \mathrm{Br}$ & (2d) & $\mathrm{C}_{3} \mathrm{~F}_{7}$ & 3d & 41 \\
\hline $\mathrm{CH}(\mathrm{OH}) \mathrm{CH}_{3}$ & $(2 \mathbf{e})$ & $\mathrm{C}_{3} \mathrm{~F}_{7}$ & $3 \mathbf{e}$ & None $^{d}$ \\
\hline $\mathrm{CH}_{2} \mathrm{CH}_{2} \mathrm{OH}$ & (2f) & $\mathrm{C}_{3} \mathrm{~F}_{7}$ & $3 f$ & None $^{e}$ \\
\hline $\mathrm{CH}(\mathrm{OAc}) \mathrm{CH}_{3}$ & $(2 \mathrm{~g})$ & $\mathrm{C}_{3} \mathrm{~F}_{7}$ & $3 g$ & 50 \\
\hline $\mathrm{CH}_{2} \mathrm{CH}_{2} \mathrm{OAc}$ & (2h) & $\mathrm{C}_{3} \mathrm{~F}_{7}$ & 3h & 83 \\
\hline $\mathrm{CH}\left(\mathrm{OSiMe}_{3}\right) \mathrm{CH}_{3}$ & (2i) & $\mathrm{C}_{3} \mathrm{~F}_{7}$ & $3 \mathbf{i}$ & 41 \\
\hline $\mathrm{CH}\left(\mathrm{OSiMe}_{2} t-\mathrm{Bu}\right) \mathrm{CH}_{3}$ & $(2 \mathrm{j})$ & $\mathrm{C}_{3} \mathrm{~F}_{7}$ & $3 \mathbf{j}$ & 28 \\
\hline $\mathrm{CH}_{2} \mathrm{CH}_{2} \mathrm{Cl}$ & $(2 \mathbf{b})$ & $\mathrm{C}_{6} \mathrm{~F}_{13}$ & $3 \mathbf{k}$ & $59^{\mathrm{b}}$ \\
\hline
\end{tabular}

a Yield was determined by GC based on the peroxides used.

b Isolated yield.

c $\mathrm{PhCH}\left(\mathrm{CH}_{3}\right) \mathrm{OCOC}_{3} \mathrm{~F}_{7}(51 \%)$ and $\mathrm{C}_{3} \mathrm{~F}_{7} \mathrm{C}_{6} \mathrm{H}_{4} \mathrm{CH}-$ $\left(\mathrm{CH}_{3}\right) \mathrm{OCOC}_{3} \mathrm{~F}_{7}(15 \%)$ were obtained.

d $\mathrm{PhCH}\left(\mathrm{CH}_{3}\right) \mathrm{OCOC}_{3} \mathrm{~F}_{7}(56 \%)$ and $\mathrm{C}_{3} \mathrm{~F}_{7} \mathrm{C}_{6} \mathrm{H}_{4} \mathrm{CH}-$ $\left(\mathrm{CH}_{3}\right) \mathrm{OCOC}_{3} \mathrm{~F}_{7}(38 \%)$ were obtained.

e $\mathrm{PhCH}_{2} \mathrm{CH}_{2} \mathrm{OCOC}_{3} \mathrm{~F}_{7}(100 \%)$ was obtained.

case of $\alpha$ - and $\beta$-phenylethylalcohols (2e, 2f), which are general styrene precursors, the hydroxy groups of $\mathbf{2 e}$ and $2 \mathbf{f}$ reacted with $\mathbf{1 a}$ to give the corresponding perfluorobutyryl esters, and the expected aromatic perfluoroalkylation was not observed. To resolve this problem, the hydroxy groups of $\mathbf{2 e}$ and $\mathbf{2 f}$ were protected by converting them to acetoxy or siloxy groups $(\mathbf{2} \mathbf{g}-\mathbf{2 j})$, which were reacted with 1a to afford $3 \mathrm{~g}-3 \mathrm{j}$ in $28-83 \%$ yield. These results are summarized in Table $\mathrm{I}$.

The perfluoroalkylation of these substrates is accounted for by the electron transfer mechanism from the aromatic nucleus of the substrates to the peroxides as was proposed previously. $^{3}$ In fact, ethylbenzene having chloro or acetoxy substituent at $\beta$-position (2b, 2h) was perfluoroalkylated in better yield than that having at $\alpha$-position $(\mathbf{2 a}, \mathbf{2 g})$ due to the inductive effect by these electron-withdrawing
Table II. Formation of perfluoroalkylated styrene

\begin{tabular}{|c|c|c|c|}
\hline \multicolumn{2}{|l|}{3} & \multirow{2}{*}{ Conditions } & \multirow{2}{*}{ Yield $^{\mathrm{a}} / \%$} \\
\hline $\mathbf{R}$ & $\mathbf{R}_{\mathrm{F}}$ & & \\
\hline $\mathrm{CH}(\mathrm{Cl}) \mathrm{CH}_{3}$ & $\mathrm{C}_{3} \mathrm{~F}_{7}$ & (3a) $\mathrm{KOH} / \mathrm{MeOH}$, refl. $4 \mathrm{~h}$ & 40 \\
\hline $\mathrm{CH}_{2} \mathrm{CH}_{2} \mathrm{Cl}$ & $\mathrm{C}_{3} \mathrm{~F}_{7}$ & (3b) $\mathrm{KOH} / \mathrm{MeOH}$, refl. $4 \mathrm{~h}$ & 66 \\
\hline $\mathrm{CH}_{2} \mathrm{CH}_{2} \mathrm{Cl}$ & $\mathrm{C}_{6} \mathrm{~F}_{13}$ & ${ }_{3}(3 \mathrm{k}) \mathrm{KOH} / \mathrm{MeOH}$, refl. $4 \mathrm{~h}$ & 77 \\
\hline $\mathrm{CH}(\mathrm{OAc}) \mathrm{CH}$ & $\mathrm{I}_{3} \mathrm{C}_{3} \mathrm{~F}_{7}$ & $(3 \mathrm{~g}) 520^{\circ} \mathrm{C}^{\mathrm{b}}$ & $46^{\mathrm{c}}$ \\
\hline $\mathrm{CH}_{2} \mathrm{CH}_{2} \mathrm{OA}$ & $\mathrm{c} \mathrm{C}_{3} \mathrm{~F}_{7}$ & (3h) $520^{\circ} \mathrm{C}^{\mathrm{b}}$ & $42^{\mathrm{c}}$ \\
\hline $\mathrm{CH}(\mathrm{OH}) \mathrm{CH}$ & ${ }_{3} \mathrm{C}_{3} \mathrm{~F}_{7}$ & (3e) $\mathrm{P}_{2} \mathrm{O}_{5} /$ Benzene, refl. $3 \mathrm{~h}$ & 95 \\
\hline $\mathrm{CH}_{2} \mathrm{CH}_{2} \mathrm{OH}$ & $\mathrm{C}_{3} \mathrm{~F}_{7}$ & (3f) $\mathrm{P}_{2} \mathrm{O}_{5} /$ Benzene, refl. $3 \mathrm{~h}$ & 0 \\
\hline
\end{tabular}

a Yield is that of the isolated product.

b Pyrolysis was carried out under a helium atmosphere.

c Yield is the conversion yield determined by GC.

groups.

We converted 3 into perfluoroalkylated styrene (4) by dehydrochlorination or dehydroacetoxylation as shown in equation 2 , and the results are illustrated in Table II.

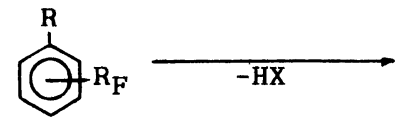

3

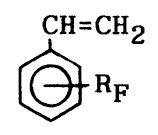

4

$$
\begin{aligned}
& \mathrm{R}=\mathrm{CHXCH}_{3}, \mathrm{CH}_{2} \mathrm{CH}_{2} \mathrm{X} \\
& \mathrm{X}=\mathrm{Cl}, \mathrm{AcO}
\end{aligned}
$$

The treatment of $\mathbf{3 a}, \mathbf{3 b}$, and $\mathbf{3 k}$ with potassium hydroxide gave perfluoroalkylated styrene in moderate to good yield. The dehydroacetoxylation of $3 \mathrm{~g}$ and $3 \mathrm{~h}$ by pyrolysis at $520^{\circ} \mathrm{C}$ formed $4 \mathbf{a}$ in moderate yield. Since perfluoroalkylated $\alpha$-phenylethylalcohol is known to be easily converted into the corresponding perfluoroalkylated styrene, ${ }^{2} \mathbf{3 g}$ and $\mathbf{3 h}$ were hydrolyzed to $\mathbf{3 e}$ and $\mathbf{3 f}$, treating with aqueous sodium hydroxide, in 87 and $64 \%$ yields, respectively. Isolated $3 \mathrm{e}$ was dehydrated by treating with phosphorus pentoxide to give $\mathbf{4 a}$ in high yield. However, $3 f$ was not converted into $\mathbf{4 a}$, and all the starting $\mathbf{3 d}$ was recovered.

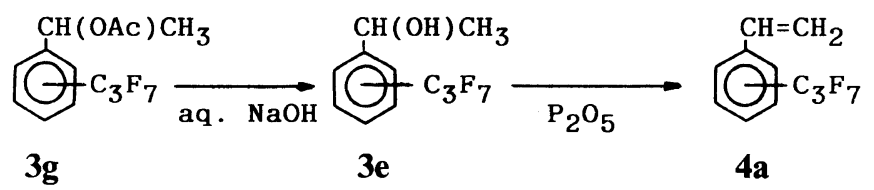


Recently, we reported the direct introduction of perfluoroalkyl groups into the aromatic nucleus of polystyrene in good yield, ${ }^{6}$ and the refractive index and glass transition temperature of this perfluoroalkylated polystyrene were found to change drastically from those of the parent polystyrene. Therefore, perfluoroalkylated styrene monomers 4 are very useful since the polymer, obtained by polymerization or copolymerization of 4 , is expected to be useful water- and oil-repellent, and applicable as optical materials in various fields.

\section{EXPERIMENTAL}

\section{Measurement}

NMR spectra were taken on a JEOL JNM Fx90Q FT-NMR spectrometer. IR spectra were recorded on a JASCO IR-810 spectrometer. Mass spectra were taken on a JEOL JMS-DX303 spectrometer by an electronimpact (EI) ionization technique at $70 \mathrm{eV}$. Gas chromatography was performed by a Shimadzu GC-8A gas chromatograph with a $2 \mathrm{~m}$ glass column (SE-30, 20\%) or a Hewlettpackard HP-5890 gas chromatograph with a $15 \mathrm{~m}$ capillary column (DB-1). Pyrolysis was performed with a YANACO GP-1018 pyrolyzer.

\section{Materials}

Bis(heptafluorobutyryl) peroxide was prepared from heptafluorobutyryl chloride and hydrogen peroxide by a method in the literature. ${ }^{7}$ Bis(tridecafluoroheptanoyl) peroxide was prepared by the same procedure.

\section{General Procedure for Perfluoroalkylation of Ethylbenzene Derivatives}

Bis(heptafluorobutyryl) peroxide (1a) or bis(tridecafluoroheptanoyl) peroxide (1b) (1.0 $\mathrm{mmol})$ in Freon 113 solution $(20 \mathrm{~g})$ were added to $2(1.5 \mathrm{mmol})$, and the solution was stirred at $40^{\circ} \mathrm{C}$ for $5 \mathrm{~h}$ under nitrogen atmosphere. Chloroform $(10 \mathrm{ml})$ was added to the reaction mixture, and the solution was washed successively with water, saturated aqueous sodium hydrogencarbonate $(20 \mathrm{ml})$, and sodium chloride $(20 \mathrm{ml})$. The separated organic layer was dried over anhydrous magnesium sulfate, and the solvent was evaporated. The residue was analyzed by GC using internal standards. The perfluoroalkylated styrene precursors (3) were isolated by distillation or preparative TLC and identified by IR, ${ }^{1} \mathrm{H}$ NMR, and GC-MS.

$\alpha$-Chloroethylheptafluoropropylbenzene (3a): IR $\left(\mathrm{cm}^{-1}\right) 1350\left(\mathrm{CF}_{3}\right) .1220\left(\mathrm{CF}_{2}\right)$; $\mathrm{MS}$ $m / z 308\left(\mathrm{M}^{+}\right), 273,153$.

$\beta$-Chloroethylheptafluoropropylbenzene (3b): bp $92-102^{\circ} \mathrm{C} / 16 \mathrm{mmHg} ;{ }^{1} \mathrm{H} \quad \mathrm{NMR}$ $\left(\mathrm{CDCl}_{3}\right) \delta=3.13(2 \mathrm{H}, \mathrm{t}, J=7.1 \mathrm{~Hz}), 3.75(2 \mathrm{H}$, $\mathrm{t}, J=7.1 \mathrm{~Hz}), 7.18-7.65 \mathrm{ppm}(4 \mathrm{H}) ; \mathrm{IR}\left(\mathrm{cm}^{-1}\right)$ $1350\left(\mathrm{CF}_{3}\right), 1225\left(\mathrm{CF}_{2}\right)$; $\mathrm{MS} m / z 308\left(\mathrm{M}^{+}\right)$, 259, 189; Exact MS: $m / z$ 308.0289, Calcd for $\mathrm{C}_{11} \mathrm{H}_{8} \mathrm{~F}_{7} \mathrm{Cl}$ : 308.0203 .

$\beta$-Bromoethylheptafluoropropylbenzene (3d): MS $m / z 352\left(\mathrm{M}^{+}\right), 273,153$.

$\alpha$-Acetoxyethylheptafluoropropylbenzene (3g): ${ }^{1} \mathrm{H}$ NMR $\left(\mathrm{CDCl}_{3}\right) \delta=1.55(3 \mathrm{H}, \mathrm{d}, J=$ $7.0 \mathrm{~Hz}), 2.08(3 \mathrm{H}, \mathrm{s}), 4.98(1 \mathrm{H}, \mathrm{q}, J=7.0$ $\mathrm{Hz}), 7.28-7.68 \mathrm{ppm}(4 \mathrm{H})$; IR $\left(\mathrm{cm}^{-1}\right) 1720$ $(\mathrm{C}=\mathrm{O}), 1350\left(\mathrm{CF}_{3}\right), 1230\left(\mathrm{CF}_{2}\right) ; \mathrm{MS} m / z 332$ $\left(\mathrm{M}^{+}\right), 153,43$.

$\beta$-Acetoxyethylheptafluoropropylbenzene (3h): bp 75-88 $\mathrm{C} / 1 \mathrm{mmHg} ;{ }^{1} \mathrm{H} \mathrm{NMR}\left(\mathrm{CDCl}_{3}\right)$ $\delta=2.06(3 \mathrm{H}, \mathrm{s}), 2.93(2 \mathrm{H}, \mathrm{t}, J=7.3 \mathrm{~Hz}), 4.30$ $(2 \mathrm{H}, \mathrm{t}, J=7.3 \mathrm{~Hz}), 7.28-7.68 \mathrm{ppm}(4 \mathrm{H})$; IR $\left(\mathrm{cm}^{-1}\right) 1780(\mathrm{C}=\mathrm{O}), 1345\left(\mathrm{CF}_{3}\right), 1230\left(\mathrm{CF}_{2}\right)$; MS (CI) $m / z 665\left[(2 \mathrm{M}+1)^{+}\right], 333\left[(\mathrm{M}+1)^{+}\right]$, 273.

Heptafluoropropyl- $\alpha$-trimethylsiloxyethylbenzene (3i): MS $m / z 362\left(\mathrm{M}^{+}\right), 347$.

$\alpha$-( $t$-Butyldimethylsiloxy)ethylheptafluoropropylbenzene (3j): ${ }^{1} \mathrm{H}$ NMR $\left(\mathrm{CDCl}_{3}\right) \delta=0.08$ $(3 \mathrm{H}, \mathrm{s}), 0.17(3 \mathrm{H}, \mathrm{s}), 1.02(9 \mathrm{H}, \mathrm{s}), 1.53(3 \mathrm{H}, \mathrm{d}$, $J=6.5 \mathrm{~Hz}), 5.03(1 \mathrm{H}, \mathrm{q}, J=6.5 \mathrm{~Hz}), 7.30-7.70$ ppm (4H); IR (cm $\left.{ }^{-1}\right) 1345\left(\mathrm{CF}_{3}\right), 1225\left(\mathrm{CF}_{2}\right)$, 1110 (SiOR); MS m/z $404\left(\mathrm{M}^{+}\right), 347,253,75$.

$\beta$-Chloroethyltridecafluorohexylbenzene (3k): bp $90-91{ }^{\circ} \mathrm{C} / 2 \mathrm{mmHg} ;{ }^{1} \mathrm{H} \mathrm{NMR}\left(\mathrm{CDCl}_{3}\right)$ $\delta=3.12(2 \mathrm{H}, \quad \mathrm{t}, \quad J=7.4 \mathrm{~Hz}), \quad 3.74(2 \mathrm{H}, \mathrm{t}$, $J=7.4 \mathrm{~Hz}), 7.21-7.65 \mathrm{ppm}(4 \mathrm{H}) ;$ IR $\left(\mathrm{cm}^{-1}\right)$ $1350\left(\mathrm{CF}_{3}\right), 1225\left(\mathrm{CF}_{2}\right) ; \mathrm{MS} m / z 458\left(\mathrm{M}^{+}\right)$, 
409, 189; Exact MS: $m / z$ 458.0102, Calcd for $\mathrm{C}_{14} \mathrm{H}_{8} \mathrm{~F}_{13} \mathrm{Cl}$ : 458.0107 .

$\alpha$-Heptafluorobutyryloxyethylbenzene: ${ }^{1} \mathrm{H}$ NMR $\left(\mathrm{CDCl}_{3}\right) \delta=1.38(3 \mathrm{H}, \mathrm{d}, J=6.5 \mathrm{~Hz})$, $4.24(1 \mathrm{H}, \mathrm{q}, J=6.5 \mathrm{~Hz}), 7.18-7.30 \mathrm{ppm}(5 \mathrm{H})$; IR $\left(\mathrm{cm}^{-1}\right) 1770(\mathrm{C}=\mathrm{O}), 1340\left(\mathrm{CF}_{3}\right), 1220$ $\left(\mathrm{CF}_{2}\right) ; \mathrm{MS} \mathrm{m} / z 318\left(\mathrm{M}^{+}\right), 105$.

1-Heptafluoropropylphenyl-1-heptafluorobutyryloxyethane: ${ }^{1} \mathrm{H} \mathrm{NMR}\left(\mathrm{CDCl}_{3}\right) \delta=1.46$ $(3 \mathrm{H}, \mathrm{d}, J=6.8 \mathrm{~Hz}), 4.52(1 \mathrm{H}, \mathrm{q}, J=6.8 \mathrm{~Hz})$, $7.20-7.40 \mathrm{ppm}(4 \mathrm{H}) ; \mathrm{MS} m / z 486\left(\mathrm{M}^{+}\right), 273$, 153.

$\beta$-Heptafluorobutyryloxyethylbenzene: ${ }^{1} \mathrm{H}$ $\operatorname{NMR}\left(\mathrm{CDCl}_{3}\right) \delta=3.03(2 \mathrm{H}, \mathrm{t}, J=7.6 \mathrm{~Hz}), 4.55$ $(2 \mathrm{H}, \mathrm{t}, J=7.6 \mathrm{~Hz}), 7.10-7.38 \mathrm{ppm}(5 \mathrm{H})$; IR $\left(\mathrm{cm}^{-1}\right) 1775(\mathrm{C}=\mathrm{O}), 1350\left(\mathrm{CF}_{3}\right), 1215\left(\mathrm{CF}_{2}\right)$; MS $m / z 318\left(\mathrm{M}^{+}\right), 104,91$.

\section{Dehydrochlorination of $\mathbf{3 a}, \mathbf{3 b}$, and $\mathbf{3 k}$}

A methanol solution $(40 \mathrm{ml})$ of potassium hydroxide $(70 \mathrm{mmol})$ was added to $\mathbf{3 a}$ or $\mathbf{3 b}$ $(75 \mathrm{mmol})$, and the solution was refluxed for $3 \mathrm{~h}$. Ether $(80 \mathrm{ml})$ was added to the reaction mixture, and the solution was washed successively with water $(100 \mathrm{ml})$, dilute hydrochloric acid $(100 \mathrm{ml})$, and saturated aqueous sodium chloride $(100 \mathrm{ml})$. The separated organic layer was dried over anhydrous sodium sulfate, and the solvent was evaporated. Heptafluoropropylstyrene (4a) was isolated by distillation under reduced pressure. Tridecafluorohexylstyrene (4b) was prepared by the same procedure from $3 \mathbf{k}$. Heptafluoropropylstyrene (4a): bp $62-65^{\circ} \mathrm{C} / 11 \mathrm{mmHg} ;{ }^{1} \mathrm{H} \quad \mathrm{NMR}$ $\left(\mathrm{CDCl}_{3}\right) \delta=5.36(1 \mathrm{H}, \mathrm{dd}, J=3.4 \mathrm{~Hz}, 10.8 \mathrm{~Hz})$, $5.82(1 \mathrm{H}, \mathrm{dd}, J=3.4 \mathrm{~Hz}, 17.5 \mathrm{~Hz}), 6.75(1 \mathrm{H}$, $\mathrm{dd}, J=10.8 \mathrm{~Hz}, 17.5 \mathrm{~Hz}), 7.20-7.70 \mathrm{ppm}(4 \mathrm{H})$; ${ }^{19} \mathrm{~F} \mathrm{NMR}\left(\mathrm{CDCl}_{3}\right)\left(\right.$ ext. $\left.\mathrm{CF}_{3} \mathrm{CO}_{2} \mathrm{H}\right) \delta=-50.4$ (2F), $-35.6(2 \mathrm{~F}),-4.5(3 \mathrm{~F})$; IR $\left(\mathrm{cm}^{-1}\right) 1350$ $\left(\mathrm{CF}_{3}\right) ; 1225\left(\mathrm{CF}_{2}\right) ; \mathrm{MS} \mathrm{m} / z 272\left(\mathrm{M}^{+}\right), 153,133$; Exact MS: $m / z$ 272.0491, Calcd for $\mathrm{C}_{11} \mathrm{H}_{7} \mathrm{~F}_{7}$ : 272.0436 .

Tridecafluorohexylstyrene (4b): bp 62$72{ }^{\circ} \mathrm{C} / 3 \mathrm{mmHg} ;{ }^{1} \mathrm{H}$ NMR $\left(\mathrm{CDCl}_{3}\right) \delta=5.34$ $(1 \mathrm{H}, \mathrm{dd}, J=3.4 \mathrm{~Hz}, 11.1 \mathrm{~Hz}), 5.80(1 \mathrm{H}, \mathrm{dd}$, $J=3.4 \mathrm{~Hz}, 17.6 \mathrm{~Hz}), 6.75(1 \mathrm{H}, \mathrm{dd}, J=11.1 \mathrm{~Hz}$,
$17.6 \mathrm{~Hz}), \quad 7.38-7.70 \mathrm{ppm}(4 \mathrm{H}) ;{ }^{19} \mathrm{~F} \quad \mathrm{NMR}$ $\left(\mathrm{CDCl}_{3}\right) \quad\left(\right.$ ext. $\left.\mathrm{CF}_{3} \mathrm{CO}_{2} \mathrm{H}\right) \quad \delta=-50.2(2 \mathrm{~F})$, $-46.7(2 \mathrm{~F}),-45.7(4 \mathrm{~F}),-35.0(2 \mathrm{~F}),-5.2$ (3F); IR ( $\left.\mathrm{cm}^{-1}\right) 1360\left(\mathrm{CF}_{3}\right), 1220\left(\mathrm{CF}_{2}\right)$; MS $m / z 422\left(\mathrm{M}^{+}\right)$, 153; Exact MS: $m / z$ 422.0288, Calcd for $\mathrm{C}_{14} \mathrm{H}_{7} \mathrm{~F}_{13}$ : 422.0340 .

\section{Hydrolysis of $\mathbf{3 g}$ and $\mathbf{3 h}$}

An aqueous solution of sodium hydroxide (70 mmol) was added to a solution in methanol $(50 \mathrm{ml})$ of $3 \mathbf{h}(70 \mathrm{mmol})$. The solution was stirred at $20^{\circ} \mathrm{C}$ for 1 day. Ether $(100 \mathrm{ml})$ was added to the reaction mixture, and the solution was washed successively with water $(100 \mathrm{ml})$ and saturated aqueous sodium chloride (100 $\mathrm{ml})$. The separated organic layer was dried over anhydrous sodium sulfate, and the solvent was evaporated. $\beta$-Heptafluoropropylphenylethylalcohol (3f) was isolated by distillation under reduced pressure. $\alpha$-Heptafluoropropylphenylethylalcohol (3e) was prepared by the same procedure from $3 \mathrm{~g}$ and isolated by preparative TLC.

$\alpha$-Heptafluoropropylphenylethylalcohol (3e): ${ }^{1} \mathrm{H}$ NMR $\left(\mathrm{CDCl}_{3}\right) \delta=1.52(3 \mathrm{H}, \mathrm{d}$, $J=7.3 \mathrm{~Hz}), 1.90(1 \mathrm{H}, \mathrm{s}), 4.97(1 \mathrm{H}, \mathrm{q}, J=$ $7.3 \mathrm{~Hz}), 7.20-7.65 \mathrm{ppm}(4 \mathrm{H})$; IR $\left(\mathrm{cm}^{-1}\right) 3350$ $(\mathrm{OH}), 1350\left(\mathrm{CF}_{3}\right), 1230\left(\mathrm{CF}_{2}\right) ; \mathrm{MS} \mathrm{m} / z 290$ $\left(\mathrm{M}^{+}\right), 275,127,43$.

$\beta$-Heptafluoropropylphenylethylalcohol (3f): bp $103-122^{\circ} \mathrm{C} / 15 \mathrm{mmHg} ;{ }^{1} \mathrm{H} \quad \mathrm{NMR}$ $\left(\mathrm{CDCl}_{3}\right) \delta=1.70(1 \mathrm{H}, \mathrm{s}), 2.92(2 \mathrm{H}, \mathrm{t}, J=$ $6.5 \mathrm{~Hz}), 3.86(2 \mathrm{H}, \mathrm{t}, J=6.5 \mathrm{~Hz}), 7.20-7.60$ ppm (4H); IR (cm $\left.{ }^{-1}\right) 3330(\mathrm{OH}), 1325\left(\mathrm{CF}_{3}\right)$, $1220\left(\mathrm{CF}_{2}\right) ; \mathrm{MS} m / z 290\left(\mathrm{M}^{+}\right), 260,141$.

\section{Dehydration of $\mathbf{3 e}$}

Phosphorus pentoxide $(18 \mathrm{mmol})$ and a catalytic amount of hydroquinone were added to a solution of $3 \mathrm{e}$ in benzene $(50 \mathrm{ml})$, and the solution was refluxed for $3 \mathrm{~h}$. Heptafluoropropylstyrene (4a) was isolated by distillation under reduced pressure from the organic layer.

\section{Pyrolysis of $\mathbf{3 g}$ and $\mathbf{3 h}$}

The pyrolysis of $\mathbf{3 g}$ or $\mathbf{3 h}$ was carried out at 
$520^{\circ} \mathrm{C}$ under helium. The pyrolyzed products were analyzed by GC-MS.

\section{REFERENCES AND NOTES}

1. K. Ishihara, R. Kogure, and K. Matsui, Kobunshi Ronbunshu, 45, 653 (1988).

2. a) V. C. R. McLoughlin and J. Thrower, Tetrahedron, 25, 5921 (1969).

b) A. Kondo, R. Haruta, T. Matsushima, and S. Iwatsuki, Polym. Prep., Jpn., 31, 805 (1982).

3. a) M. Yoshida, H. Amemiya, M. Kobayashi, $\mathbf{H}$. Sawada, H. Hagii, and K. Aoshima, J. Chem. Soc.,
Chem. Commun., 234 (1985).

b) H. Sawada, M. Yoshida, H. Hagii, K. Aoshima, and M. Kobayashi, Bull. Chem. Soc. Jpn., 59, 215 (1986).

4. M. Yoshida, K. Moriya, H. Sawada, and M. Kobayashi, Chem. Lett., 755 (1985).

5. The product is found to be a mixture of three isomers (probably ortho, meta, and para) by GC analysis with a capillary column.

6. H. Sawada, M. Mitani, M. Nakayama, M. Yoshida, and N. Kamigata, Polym. Commun., 31, 63 (1990).

7. C. Zhao, R. Zhou, H. Pan, X. Jin, Y. Qu, C. Wu, and X. Jiang, J. Org. Chem., 47, 2009 (1982). 